\title{
LAS CONCEPCIONES DE LA LECTURA Y LA ESCRITURA EN LA LABOR DEL DOCENTE DE EDUCACIÓN SUPERIOR
}

\begin{abstract}
Resumen
El objetivo de esta investigación consiste en "identificar las concepciones de los docentes acerca de la lectura y de la escritura como herramientas pedagógicas en el desempeño académico universitario". El proceso investigativo es de carácter exploratorio descriptivo, intenta identificar las concepciones de la lectura y la escritura en la dinámica de los procesos de enseñanza aprendizaje en la Facultad de Educación de la Universidad de La Salle. La propuesta se integra dentro del desarrollo de la línea de Investigación en Pedagogía y Didáctica.

Palabras claves: Lectura, escritura, concepciones, enseñanza, aprendizaje, práctica pedagógica.

\section{THE CONCEPTIONS OF THE READING AND THE WRITING IN THE LABOR OF THE} TEACHER OF HIGH EDUCATION
\end{abstract}

\begin{abstract}
The objective of this investigation consists of "identifying the conceptions of the educational ones about the reading and the writing like pedagogical tools in the university academic performance". The investigative process is of descriptive exploratory character, tries to identify the conceptions of the reading and the writing in the dynamics of the education processes learning in the Faculty of Education of the University of the Salle. The proposal Integra within the development of the line of Investigation in Pedagogy and Didactic.
\end{abstract}

Keywords: Reading, writing, conceptions, education, learning, pedagogical practice.

* Magister en literatura. Candidato a Doctor en Sociedad de la información y el conocimiento. Profesor de la Universidad Pedagógica Nacional, Bogotá. rmedina@pedagogica.edu.co

** Magíster en lingüistica hispánica. Profesora Universidad Pedagógica Nacional. Lilica2002@mixmail.com 


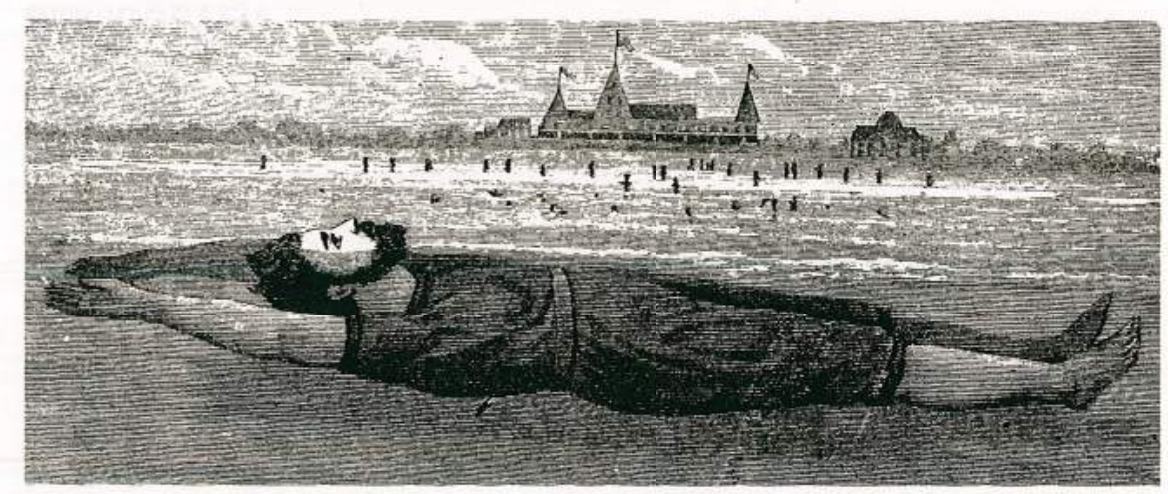

\section{INTRODUCCIÓN}

Este artículo surge del proyecto de investigación titulado "Las concepciones y las prácticas de lectura y de escritura en la labor del docente de la Facultad de Educación de la Universidad de La Salle". Como hipótesis de trabajo se consideró que los docentes poseen sus propias concepciones en relación con los procesos de la lectura y la escritura. Estas concepciones se manifiestan a través de las formas y los procedimientos didácticos que asumen en los procesos de enseñanza de las disciplinas.

Para los fines de este estudio se empleó una metodologia de naturaleza cualitativa con un diseño de tipo descriptivo básico. Como referentes conceptuales se tomaron, en la medida que conforman modelos explicativos de la lectura y de la escritura y movilizan los conocimientos del docente para adaptarlos al acto pedagógico, las aproximaciones teóricas elaboradas por autores como Huey (1908), Thorndike (1917) y Gray (1937) en la concepción tradicional; Rosemblatt (1938), en la concepción transaccional; Smith (1980) y Goodman (1982), en la concepción psicolingüistica; Jaimes (1994), en la concepción psicosociolingüistica, y De Zubiria (1997) en la concepción cognitiva. Los planteamientos de estos autores se reconocen en las concepciones epistemológicas de los docentes y, a su vez, posibilitan identificar creencias e ideas acerca de la lectura y la escritura expresadas en enunciados que representan redes semánticas y pragmáticas de conocimientos acumulados y explicitados en procedimientos y actos didácticos.

\section{METODOLOGÍA}

Se asumió una metodología de naturaleza cualitativa con un diseño de tipo descriptivo. Este enfoque metodológico permitió trabajar sobre realidades existentes y proporcionó una descripción detallada de una serie de eventos, comportamientos, situaciones y personas observables, alli se incorporó la voz de los participantes, sus creencias, experiencias, pensamientos, actitudes y reflexiones tal y como ellos mismos la expresan. De esta forma se generó una interpretación adecuada de la naturaleza de las cosas, personas, grupos, procesos y acontecimientos de una manera amplia y objetiva sin entrar a intervenir el objeto estudiado para modificarlo.

Para su desarrollo, en el proceso de observación inicialmente se definió la población: los docentes de la Facultad de Educación de la Universidad de La Salle; luego, para la identificación de las concepciones de la lectura y la escritura, se emplearon tres tipos de instrumentos: entrevistas, encuestas y observación participante. Los datos obtenidos fueron categorizados y analizados de forma separada. Para efectos de análisis, se clasificaron en: registros de orden conceptual, se recogieron las opiniones conceptuales frente a los modelos, los enfoques y las teorías referentes a los procesos de lectura y escritura; registros de tipo metodológico, se recolectó la información correspondiente al conocimiento de las metodologías de la enseñanza de los procesos de lectura y de escritura; y registros de tipo reflexivo, se compilaron las reflexiones acerca de la importancia y el sentido que le otorga el docente a la lectura y la escritura en el día por día, en el trabajo de aula.

Con base en los criterios de clasificación de la información se definieron las siguientes dimensiones:

1. Formación conceptual de los docentes frente a los procesos de la lectura y la escritura: conocimientos acerca de los modelos teóricos y epistemológicos relacionados con la lectura y la escritura.

2. Relación entre el saber teórico y el desempeño metodológico del docente: la aplicación de las metodologías de la enseñanza de las disciplinas y el soporte teórico de estas metodologías en la lectura y la escritura.

3. Desarrollo de los procesos de lectura y escritura en su práctica docente: las formas como el docente es usuario de los procesos de lectura y de escritura en su práctica laboral.

Estas dimensiones se analizaron con un enfoque descriptivo con el fin de caracterizar las concepciones de los docentes.

\section{RESULTADOS}

\section{Primera dimensión}

Una característica dominante de las opiniones de los profesores en relación con los procesos de lectura y de escritura es el desconocimiento de teorías y de enfoques epistemológicos y conceptuales existentes sobre estos procesos. Por tanto, se evidencia que las diversas voces de los maestros referencian opiniones dispersas y despojadas de referentes teóricos relacionados con los procesos de lectura y de escritura. Sobre las preguntas que conducían a identificar cuál es concepción de lectura y escritura de los docentes de la facultad, las respuestas manifestaron opiniones que adolecen de una postura epistemológica. El $81 \%$ de las respuestas son consideraciones personales que no se encuentran soportadas en algunas corrientes de pensamiento o en algunas tendencias o enfoques teóricos 
que trabajan al respecto; en tal sentido, sobresalen expresiones como

"La lectura es un proceso de decodificación de signos escritos para darles significado, reelaborarlo e interpretarlo. Así como la escritura es un proceso de plasmar ideas con símbolos del alfabeto de una lengua", "La lectura y la escritura son actividades eminentemente intelectuales y prácticas, las cuáles nos permiten comunicarnos con los demás, concentrarnos en el pensamiento y el sentir de culturas antiguas, pero también, en las mentes lúcidas del presente".

Asi mismo, existen docentes que no establecen diferencia entre la lectura y la escritura, las consideran inseparables e indisolubles: "La lectura y escritura nos instruyen y nos conducen a capacitarnos para llevar una vida plenamente humana tanto a nivel personal como colectivo", "La lectura y la escritura son métodos de aprendizaje para llegar a la interpretación de un texto por medio de diferentes signos con un aprendizaje significativo de acuerdo a la edad y procesos llevados".

Ahora bien, sobre el conjunto de preguntas que posibilitaban identificar las teorias sobre la lectura y la escritura que conocen y con cuáles se identifican, es importante señalar que el $12.7 \%$ de los docentes, manifestaron conocer algunas teorias sobre la lectura y la escritura. Dentro de este grupo, el $20 \%$ hace referencia a teorias y planteamientos teóricos diferentes al enfoque comunicativo, el $80 \%$ restante menciona que conoce y utiliza el enfoque comunicativo en la práctica docente que realiza, es decir, el $87.3 \%$ de los profesores desconoce desarrollos teóricos y epistemológicos sobre los procesos de lectura y de escritura; al respecto algunas voces manifiestan:

"yo no conozco ningún modelo, sin embargo, me identifico con el constructivista cognitivo porque se considera el desarrollo cognitivo y el proceso de construcción que se logre por parte de cada lector", "la verdad, no conozco teoria especíica al respecto "No, en verdad no he pensado en estudiar teorias de la lectura y la escritura; pues también uno da por hecho que el muchacho en la universidad sabe leer y escribir".

Frente a los docentes que manifiestan el conocimiento del enfoque comunicativo es reiterada las respuestas como: "yo trabajo el enfoque comunicativo. porque tanto escritura y escritura son habilidades comunicativas", "si conozco el enfoque comunicativo, qué como lo explico, bueno porque el lenguaje es comunicación, con la escritura y la lectura nos comunicamos, interactuamos con los demás", "creo en el enfoque comunicativo porque dá buenos resultados, en la enseñanza de las lenguas extranjeras es la mejor metodología" "me ha gustado la teoria de las seis lectura del Merani, pero normalmente se considera que un estudiante universitario está en capacidad de leer y escribir bien, ah, también el texto de Zuleta, ese es un abc que debe conocer cualquier docente".

De otro lado, un caso bastante significativo giró alrededor de la pregunta que exploraba sobre el conocimiento, la caracterización y la diferencia de las tipologias textuales, pues este evidenció que el $14 \%$ de los profesores tiene claridad sobre las tipologias textuales, mientras que el restante $86 \%$ no explicita una tipología coherente con los enfoques lingüisticos y discursivos ya que en la mayoría de las respuestas hacen referencia a clasificaciones identificadas con los dominios disciplinares; veamos algunas de las respuestas:

"frente a las tipologías diferencio la narrativa, la lírica, el teatro y, ensayo", "bueno yo trabajo de la siguiente manera, yo clasifico los textos entre libros y ensayos", "mire yo trabajo con libros y artículos cientificos, a veces utilizo alguna pelicula como elemento adicional, pero toda la literatura que se trabaja es cientifica, ese el tipo de texto que trabajo",

\section{Segunda dimensión}

Es importante destacar la existencia de limitaciones conceptuales en torno a los núcleos del saber pedagógico, especialmente los referidos a la enseñabilidad de las disciplinas y a la educabilidad de las mismas. Las afirmaciones de los entrevistados posibilitaron reconocer que no existen categorias pedagógicas (metodologías de la enseñanza de las diferentes disciplinas: Matemáticas, Teología, Biología, Semántica, Literatura, Filosofia, etc.) en los desempeños de la mayoria de los docentes, es decir, en un $78 \%$, mientras que en el $22 \%$ restante el conocimiento de las metodologías de la enseñanza de las disciplinas no ha sido suficientemente interiorizado, lo que contribuye a un distanciamiento entre la relación de los dominios disciplinares con los procesos de lectura y de escritura como factores determinantes en el proceso de enseñanza aprendizaje.

Por lo general, los docentes manifiestan que no establecen relaciones entre las prácticas de lectura y de escritura con estrategias y procedimientos pedagógicos y didácticos que determinan la dinámica del proceso de enseñanza aprendizaje. Al consultar a los docentes frente a las preguntas que buscan identificar el sentido pedagógico y didáctico con que los docentes utilizan los procesos de lectura y de escritura, asi como la intención pedagógica y didáctica cuando se solicita la elaboración de textos, resúmenes, informes, comentarios, ensayos o reseñas, el $98 \%$ de los docentes manifestó que nunca habia pensado esa relación. Esta consideración, puede resumirse en una de las respuestas que condensa la reiteración significativa de los entrevistados:

"en verdad yo no he concebido ese tipo de relación, simplemente pido la elaboración de los textos para evidenciar que los muchachos leyeron los textos y que pueden expresar ideas concernientes con los temas tratados". Otros docentes manifiestan que: "el valor pedagógico del texto se lo dá el maestro, yo pido que elaboren ensayos porque ellos tienen que aprender a escribir"

Un aspecto que llama la atención es que el $80 \%$ de los docentes afirma que es constante, en su práctica pedagógica, abordar manuales que sintetizan los contenidos teóricos de las diferentes disciplinas y áreas del conocimiento, es decir 
se utilizan textos "resumen" para trabajar en las sesiones. Quienes emplean estos materiales argumentan que:

"Ios utilizo porque el espacio de las sesiones me lo permite, pero también porque creo que estos textos eventualmente pueden acercar más y mejor los temas", "no hay tiempo para abordar las fuentes originales" "a los estudiantes no les gusta y les parece muy complejo leer algunas obras y autores" "no existe la cultura dentro de nuestros estudiantes de leer las obras completas y los textos propios de cada autor, por lo tanto, lo que se leen son las versiones de comentaristas de los grandes autores".

Coinciden en afirmar que la recepción de los textos por parte de los estudiantes no es la mejor, normalmente es catalogada como "aceptable" porque "no tienen intereses especiales"; "es muy irregular", "el cansancio influye, sobre todo en los estudiantes de la jornada nocturna"; "se lee a medias, a la carrera y además hay lecturas que siendo atentas no cuentan con referentes significativos para el lector"; "rechazan los textos que no entienden rápido. Otros rechazan (...) sin buscar otras dimensiones de lo científico o lo técnico, lo estético y lo poético".

Frente a la dinámica de clase, en relación con la forma como se desarrolla la sesión, los docentes destacan que la clase está apoyada en el diálogo entre los participantes del encuentro; por tanto, la dinámica depende de la lectura previa de los estudiantes y el profesor fomenta la participación de los alumnos. Se considera pertinente que se compartan en la facultad unas orientaciones metodológicas básicas para dirigir los cursos.

Frente al grupo de preguntas que conducen a identificar los criterios pedagógicos con los cuales los docentes utilizan los procesos de lectura y de escritura en su práctica, se hace referencia a la forma como se trabajan los textos que se leen para desarrollar los contenidos de los espacios académicos; sin embargo, no expresan ningún enunciado que evidencie las apuestas pedagógicas y didácticas de asumir la lectura y la es- critura; en otras palabras, no se piensa sobre el valor pedagógico y didáctico que tiene un resumen, una reseña, un comentario o un ensayo, tanto en el sentido de su producción como en el de lectura. De igual manera, se hace explicita esta apreciación frente al encuentro con los libros, en donde además se advierte que en la mayoria de los casos no se trabajan en su totalidad sino, por lo general, capítulos aislados que hacen referencia al tema específico que está desarrollando.

Sin embargo, frente al número de preguntas que exploraban la importancia que le otorga el docente a los procesos de lectura y de escritura en la asimilación y la construcción de los conocimientos específicos de su asignatura el $100 \%$ de los profesores coincide en que el aprendizaje se sustenta en la lectura y, al mismo tiempo, evidencian que los estudiantes tienen deficiencias en la comprensión e interpretación de los textos.

En relación con la escritura, los docentes afirman que existe un manejo precario del proceso de escritura, se insiste en el descuido de la ortografía, de la redacción, en el escaso vocabulario que conoce un estudiante universitario y en la composición de los textos escritos; también se resalta el uso inadecuado de Internet, en donde se destaca que no se citan los autores, se asumen como propios los textos tomados de la red $y$, tampoco se verifica la calidad de la información.

De otro lado, algunos docentes manifiestan que el problema del conocimiento no es el acceso a la información; consideran que tanto la lectura como la escritura son medios para acceder a la información, pero el proceso de la asimilación del conocimiento está relacionado más con los aspectos psíquicos que con los informativos.

Frente a las preguntas orientadas a identificar las formas como los docentes determinan los tipos de textos utilizados en el proceso de enseñanza aprendizaje, los docentes afirmaron que en muy pocas ocasiones se piensa en las calidades pedagógicas de los textos. Por lo general se afirma que "de acuerdo con el nivel cognitivo de los estudiantes, el espacio académico y el grado de complejidad que se requiera. En ocasiones, según el gusto de los estudiantes".

De igual modo, en relación con los interrogantes dirigidos determinar el tipo de texto que el docente propone para lectura: ensayos, libros, artículos científicos, reseñas, entre otros; y el tipo de producciones textuales exigidos para su desempeño académico, el $95 \%$, manifiesta que la escogencia de los textos depende de los contenidos que se trabajan en la clase. Por lo general, "los estudiantes tampoco se preocupan por si la lectura es un ensayo, o un libro o un artículo científico".

\section{Tercera dimensión}

La producción textual de los docentes realmente es mínima, el $89.5 \%$, expresa que no tiene tiempo para escribir, para producir textos académicos como ensayos, artículos libros o reseñas publicables. La gran mayoría, el $98 \%$, sostiene que sus escritos se encuentran orientados más hacia la preparación de clases y a responder con documentos administrativos que se exigen desde los programas académicos; es decir, que frente a los procesos de escritura que realizan los profesores, estos manifiestan que en realidad su producción se fundamenta en textos funcionales tales como correspondencia, informes memorandos $y$ actas que se elaboran en las reuniones.

Frente a la lectura afirman que la lectura que más realizan se relaciona con las fuentes bibliográficas que se trabajan en las clases; es decir, se lee para la preparación de las clases, es muy poca la lectura lúdica o recreativa que realizan y el ciento por ciento destaca que realiza un tipo de lectura "informativa, fundamentalmente periódico para enterarse de la realidad".

Sin embargo, en el conjunto de interrogantes que tenian como objetivo identificar el tipo de textos que lee y produce el docente en su vida personal y profe- 
sional, son muy pocos quienes afirman que han producido artículos científicos 0 reseñas publicadas en revistas o libros. En torno a la escritura manifiestan:

"escribir es sumamente dificil, hacer algo importante es complejo y requiere de tiempo, que es justamente lo que no tenemos, por eso no escribimos, claro que eso no es una justificación", "prefiero leer que escribir, me sirve más en el trabajo.", "publicar cada vez es más fácil; pero a la vez más complicado si se piensa decir algo nuevo, por eso los docentes preferimos leer", "la escritura es compleja, como maestro reconozco que si nos hace falta escribir más. Uno prepara sus apuntes de clase; pero ahí no hay redacción, no hay composición, hay ideas sueltas; es decir no hay un documento bien armado".

Son muy pocos los docentes que aseguran tener algún tipo de texto publicado (libro ensayos, artículos o textos literarios). Este escaso grupo, reitera las dificultades de tiempo y condiciones necesarias para la práctica de la escritura.

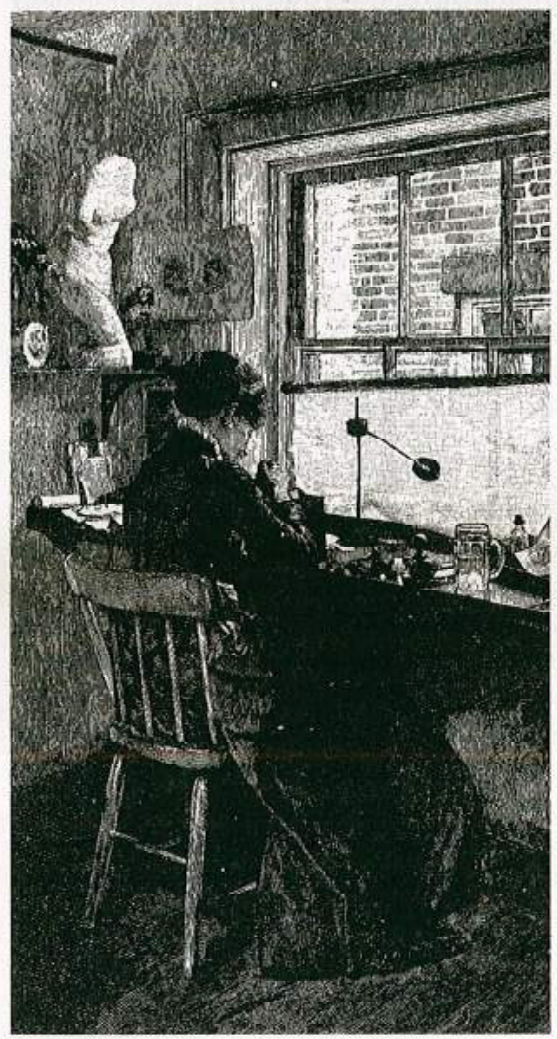

\section{DISCUSIÓN}

En cuanto a la formación conceptual de los docentes frente a los procesos de la lectura y la escritura, asumir los procesos de lectura y de escritura como un reconocimiento de significantes y referentes textuales sin identificar el orden del discurso es la concepción de mayor predominio entre los profesores de la Facultad. En esta concepción, los profesores consideran que los estudiantes tienen pleno conocimiento del universo de las palabras -el léxico puesto en referentes-que aparecen en un texto: el estudiante sabe o conoce el significado de cada término y la suma de los significados permite al estudiante comprender - explicitar ideas en los momentos de lectura o de escritura.

En este sentido, tanto la comprensión (lectura, según denominan los maestros) como la representación (escritura) se toman como un conjunto de habilidades que posee el estudiante para comprender o explicitar lo referenciado en un texto. Por tanto, esta concepción se inspira en el reconocimiento de la representación grafofonémica donde se establece y predomina un carácter sintagmático en las unidades léxicas y oracionales que componen un texto y donde el significante posee un carácter fundamental, tanto en la lectura como en la escritura; es decir, esta concepción se basa en el desciframiento y ciframiento del código de la lengua. Esta característica identifica la lectura y la escritura como un sistema de habilidades de carácter factoperceptible; concepción que coincide con el modelo tradicional (Gray, 1937) en donde se considera al texto como unidad de análisis y como unidad de creación.

Los profesores identifican la lectura y la escritura como productos derivados de un proceso donde las partes de un texto se dividen en el momento de leerlo o de escribirlo. Esta consideración determina que tanto la lectura como la escritura se dinamicen a través de las reglas establecidas por el aparato gramatical del sistema de la lengua, el significado del texto se produce gracias a la movilidad de dichas reglas. Desde este criterio, sólo se reconoce para la lectura y la escritura un carácter inductivo en los momentos de producción y de recepción; aquí su configuración se realiza en jerarquias que parten de las nociones, transcurren por los conceptos y continúan hacia las categorías. En este sentido, vale la pena recordar que en los procesos de lectura y escritura intervienen niveles de contextualización, paratextualización, interxtualización así como los sincretismos, los interdiscursos y las citas entre otros elementos propios de los procesos deductivos que hacen posible la convergencia productiva de texto en ausencia de contexto o de producir contexto a partir del texto.

De este modo, se evidencia una perspectiva sintagmática o lineal que estrecha los procesos de lectura y de escritura a la dimensión textual. Por consiguiente, las implicaciones didácticas y pedagógicas que se producen proyectan un ejercicio pedagógico dirigido hacia el cuidado normativo y correctivo de las unidades léxicas y oracionales de los textos y se desatienden aspectos relacionados con la riqueza de los contenidos y la interrelación de los contextos.

Por esta razón, este modelo se sustenta en el uso pedagógico y didáctico de la lectura y la escritura como medios que favorecen la transferencia de información en el proceso de enseñanza aprendizaje. Concepción en la que se asume la acción sistematizadora de entrenamiento del sistema de habilidades de carácter sensoromotrices para perfeccionar los niveles de lectura y de escritura; es decir, su énfasis recae en realizar una lectura o escritüra a una velocidad considerable, manteniendo el uso correcto de la gramática, reconociendo el significado unívoco y preciso de cada referente y centrando su atención en el sistema de la lengua para reconocer, únicamente, el nivel textual a partir de las unidades léxicas y oracionales.

De esta manera, el aprendizaje del estudiante se proyecta desde una perspectiva didáctica de asistencialidad y de dependencia del profesor. Implica 
un procedimiento que requiere la intervención directa del docente para lograr la enseñanza, se otorga una amplia prioridad a los elementos lógicos y técnicos de la lengua desde una dimensión sintética. Así, el modelo resulta centrado más hacia la enseñanza que hacia el aprendizaje porque los procesos de la lectura y la escritura demandan un procedimiento sistemático de la acción educadora del docente para hacer evidente el universo contextual y discursivo de la lectura y la escritura, -esta concepción puede entenderse como una extensión de métodos de lectura como el alfabético, el silábico, el onomatopéyico, el fonético, el de palabras generadoras, el global, el idovisual y el ecléctico o mixto, utilizados para desarrollar procesos de adquisición de lectura y de escritura en niños recién escolarizados.

En cuanto a la relación entre el saber teórico y el desempeño metodológico del docente, en la Universidad se insiste en el reconocer y validar la tradición pedagógica de san Juan Bautista de La Salle, fundamentada en la autoridad del maestro y en la tendencia informativa y memorística de la enseñanza. Desde esta concepción, los procesos de la lectura y la escritura se fundamentan en la comprensión literal o habilidad para comprender lo explícitamente dicho en el texto, (Dubois, 1991). Sin embargo, las directivas de la Facultad manifiestan asumir el modelo lasallista; pero, tratan de incorporar una concepción de pedagogía crítica, que resulta totalmente opuesta a la concepción lasallista. Unido a esto, se exige a los docentes hacer énfasis en el desarrollo de competencias y en la formación de habilidades y destrezas. Por tanto, se evidencia una desorientación epistemológica y conceptual sobre las implicaciones que tienen los procesos de la lectura y la escritura en las prácticas pedagógicas determinadas por los modelos pedagógicos que se asuman en la enseñanza.

Así mismo, existe una preocupación de los docentes de la Facultad de Educación por aplicar pedagogias no tradicionales en el marco de los espacios académicos correspondientes, en tanto que insisten en el reconocimiento y la afi- liación a diversos modelos pedagógicos, fundamentalmente de corte constructivista; pero se desconoce que en estos modelos los procesos de la lectura y la escritura se caracterizan porque se asumen como procesos interactivos en donde el usuario construye el sentido del texto; es decir, la compresión y la producción textual no se realiza en el reconocimiento de las palabras o de las letras de manera literal sino en el énfasis otorgado al significado gracias a las experiencias y los conocimientos que ya posee el usuario (Smith, 1980; Goodman, 1982). Por consiguiente, aunque los profesores tratan de asimilar una perspectiva pedagógica que les permita cualificar su práctica docente con el fin de superar el abordaje metodológico tradicional, las posturas pedagógicas asumidas resultan nutridas por la concepción tradicional de la lectura y la escritura. Esta consideración ilustra un amplio desconocimiento de la importancia que tiene la comunicación, la acción humana (social) y el lenguaje -la lectura y la escritura-en relación con los procesos formativos, de carácter pedagógico y didáctico, que plantean las perspectivas pedagógicas contemporáneas.

Esta evidente contradicción determina una serie de implicaciones que alteran un desarrollo armónico de la actividad académica de la Facultad soportada en la lectura y la escritura como elementos pilares en el proceso de formación profesional de los estudiantes.

De otro lado, son pocos los docentes que manifiestan conocer las teorias contemporáneas sobre los procesos de la lectura y la escritura. Este grupo de profesores evidencia que los estudios actuales sobre la lectura y la escritura han cobrado un reconocimiento determinante debido a la importancia que se les asigna en los procesos de aprendizaje y su influencia sobre el éxito o fracaso escolar. No obstante, expresan que no tienen en cuenta el conocimiento de dichas teorias en el desarrollo de la dinámica del proceso de enseñanza aprendizaje de su práctica laboral. Por tanto, el abordaje de estos conocimientos se asume como un tema o contenido específico para los estudiantes de len- guas. De manera curiosa, este grupo de docentes afirma que la aplicación de los modelos y teorias sobre la lectura y la escritura cobran trascendencia cuando se les enseña a leer y escribir a los niños de educación básica, por lo que descartan la utilización de dichas teorias en la educación universitaria.

En el caso de los profesores de la Licenciatura en Lengua Castellana, Inglés y Francés, manifiestan que conocen y aplican el enfoque comunicativo para la enseñanza de la segunda lengua. Sin embargo, en sus planteamientos se desconoce que para el caso de la enseñanza de la lengua, el enfoque comunicativo tiene como objeto la enseñanza de la lengua como comunicación significativa, ya que el enfoque comunicativo ha surgido gracias a la confluencia de diversas disciplinas como la filosofía analítica o pragmática filosófica, la antropologia lingüistica y cultural, la sociolingüística, la psicologia cognitiva y, especialmente, los enfoques discursivos y textuales, (Lomas y Osorio, 1993). Este desconocimiento, hace que los docentes asuman una perspectiva inversa al enfoque comunicativo, centrada en el aprendizaje y en el manejo de la gramática, fortalecida en el enriquecimiento lexical y con una fuerte insistencia en el estudio de la lengua como un sistema exclusivamente gramatical. Esta perspectiva pone en evidencia una fuerte confusión entre los conceptos de lengua y lenguaje, de lengua y habla, de texto y discurso y, de texto y contexto, entre otros aspectos que determinan la complejidad de los procesos de la lectura y la escritura

Estas consideraciones implican una reducida concepción del enfoque comunicativo que limita la enseñanza y el aprendizaje de la lectura y la escritura porque las enmarca en el estudio de la dimensión gramatical y en el uso correcto de la norma; es decir, una enseñanza centrada en el correcto escribir y leer desde la perspectiva gramatical en detrimento de la actividad del lenguaje desencadenada en la producción de los factores que surgen en la praxis sociocultural y que se revela en los procesos de la lectura y la escritura como construcción social. 
El hecho de no asumir el estudio del lenguaje en sus distintas manifestaciones aleja las posibilidades de abordar la lectura y la escritura desde una perspectiva interdisciplinaria donde convergen aspectos de orden cognoscitivo, discursivo y cultural que se encuentran coarticulados indefinidamente en los textos y en los discursos.

Como no se considera el enfoque comunicativo desde la integralidad de su naturaleza, no se conciben los procesos de la lectura y la escritura como capacidades humanas de naturaleza social que cumplen una función de gran importancia en el desarrollo de la cognición y de la conciencia social de los individuos en el proceso de enseñanza aprendizaje. Esta caracteristica evita comprender que la lectura y la escritura son sistemas semióticos de mediación en la configuración del sentido, a partir de realidades naturales y sociales, mediados por aspectos cognoscitivos, sociales y culturales que se interrelacionan en la construcción de la significación; por tanto, lastimosamente no se reconoce que la lectura y la escritura son portadoras de un carácter interdisciplinario y totalizante que integran las actividades representativa (cognoscitiva), interactiva y estética en el marco de la práctica sociocultural de la acción del hombre sobre el mundo y sobre los demás.

En este sentido, se evidencia la necesidad de asumir los procesos de la lectura y la escritura más allá del componente gramatical; es decir, reconocerlos como sistemas significativos y actuativos ya que son procesos de interacción comunicativa que surgen en la acción social. Concepción que implica superar los rasgos morfosintácticos y fonológicos que cimientan la lectura y la escritura para asumir el reconocimiento de las relaciones existentes entre la lengua y los contextos socioculturales que destacan el carácter sistémico de la lengua en cuanto a su estructura y funcionamiento y, de la misma forma, traer a escena las concepciones de las teorías de la significación, los desarrollos de la semántica y las formas como operan dichas teorías en la construcción de la significación, estas consideraciones dimensionan los procesos de la lectura y la escritura como sistemas de producción de sentido.

Otro de los aspectos se relaciona con el hecho de no abordar las teorías de la pragmática, la enunciación y el análisis del discurso como un universo teórico que supera la lingüistica oracional en el proceso de enseñanza-aprendizaje. En tal sentido, la orientación sobre los procesos de la lectura y la escritura se mantiene en una perspectiva oracional como producción y expresión de la conciencia social en donde también se omite los desarrollos de la semántica y de las teorias de la significación.

Como consecuencia, la atención se ha centrado en la unidades oracionales y no en las discursivas, se han descuidado los aspectos pragmáticos y retóricos manifiestos en los actos comunicativos de la escritura y la lectura; es decir, se ha negado el dominio de los procedimientos que ofrece el lenguaje para organizar la significación y actuar de acuerdo con los propósitos del discurso por medio del conocimiento del sistema formal, significativo y actuativo de la lengua.

Otro aspecto relevante está relacionado con la insistencia en el conocimiento de la lengua para comunicar y no en el estudio del lenguaje como comunicación. Situación que recae en el privilegio de concebir la lectura y la escritura como un conjunto de habilidades que operan para la transmisión de información en el proceso de enseñanza aprendizaje y se desconoce que el enfoque comunicativo, para los procesos de enseñanza de la lectura y la escritura, se encuentra articulado a la vida cultural de la expresión humana; es decir, la enseñanza de la lectura y la escritura debe estar vinculada más al uso del lenguaje que al de la lengua; por tanto, no acepta niveles de instrumentalización ni del desarrollo de habilidades o destrezas en la medida que estas resultan ser componentes aleatorios, pero no definitivos en el desarrollo de la escritura y la lectura.

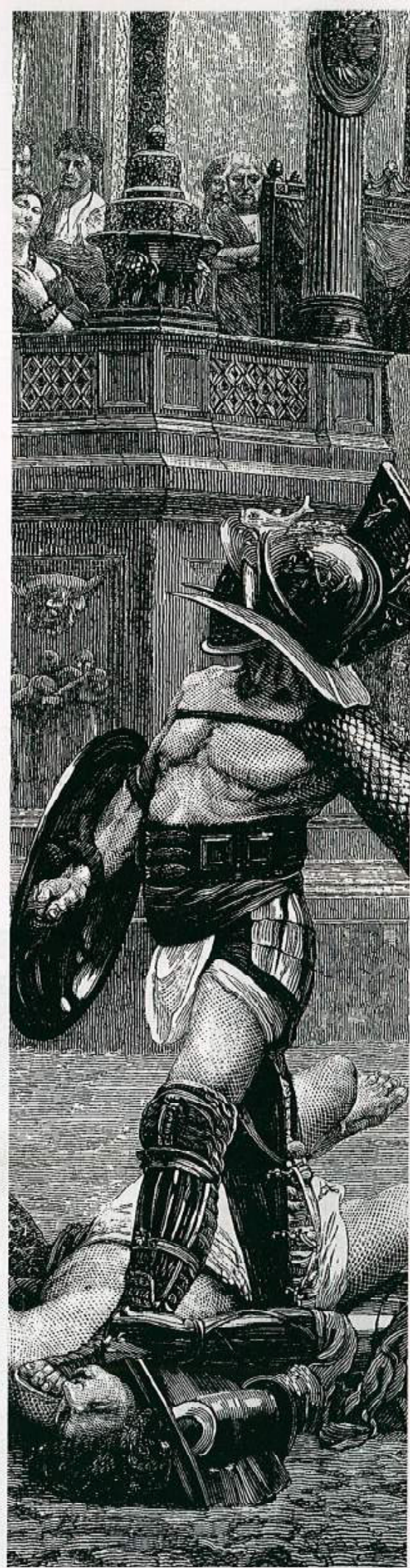




\section{CONCLUSIONES}

Este estudio identifica las concepciones de los maestros de la Facultad de Educación de la Universidad de La Salle acerca de los procesos de la lectura y la escritura. Se evidencia el dominio de una concepción tradicional, tanto de la enseñanza como de los mismos saberes específicos de los procesos de la lectura y la escritura. En cuanto a la forma como se reflejan estas concepciones en las acciones didácticas en el aula, el énfasis recae sobre el conocimiento estructural del componente lingüístico; es decir, en la práctica de fórmulas de redacción, de composición y de estructuración gramatical. En este sentido, las concepciones que se revelan, destacan fundamentalmente su dimensión instrumental en el proceso comunicativo y el no reconocimiento de los procesos de la lectura y la escritura desde una perspectiva que explique los procesos de elaboración y configuración de sentido en el contexto de una praxis social y cultural como lo es el proceso de enseñanza aprendizaje, en donde a su vez los procesos de la lectura y la escritura son uno de los dispositivos esenciales para el aprendizaje.
También se identifica una concepción normativa de la lengua escrita que obstaculiza la construcción de sentido en los procesos de la lectura y la escritura en diversas prácticas académicas. Esta mirada se convierte en un indicador que determina el porqué de las acciones y de las orientaciones desarrolladas por los docentes en su práctica pedagógica. Manifiestan una relación estrecha entre los comportamientos del docente frente a los estudiantes y los saberes desarrollados en las diversas disciplinas, a través de la conciencia discursiva en la acción pedagógica.

La forma como el docente emplea la lectura y la escritura en su práctica docente, y la manera cómo se reflejan estas concepciones en las acciones didácticas en el aula se encuentran determinadas por una didáctica implícita de carácter subjetivo y dependen de la autonomía del maestro; por tanto, los docentes adoptan algunos rasgos generales que limitan una suficiencia teórica que, de hecho, repercute en la práctica docente. Del mismo modo, el afán de asumirse dentro de un modelo pedagógico genera tensiones de desencuentro en las relaciones establecidas entre los modelos pedagógicos y sus desarrollos ligados a los procesos del lenguaje, dentro de ellos, la lectura y la escritura.

Ante esta situación emerge para la Facultad la necesidad de configurar y unificar una postura epistemológica y metodológica en relación con los procesos de la lectura y la escritura, pues no es ajeno plantear que en el ámbito académico universitario no es frecuente develar las concepciones sobre la enseñanza y el aprendizaje, en este caso particular, de la lectura y de la escritura. Aunque, como prácticas académicas, se encuentren implícitas, repercutan en el proceso de construcción del conocimiento y determinen la práctica pedagógica del docente. La ausencia de su explicitación no favorece la reflexión acerca de la naturaleza de estos procesos y del reconocimiento de la multiplicidad de especificaciones que se integran en los procesos de construcción del conocimiento. Además, se hace importante la movilización de espacios de actualización donde se propicie el acercamiento y la discusión a las teorías que la comunidad académica ha desarrollado sobre los procesos de la lectura y la escritura para establecer relaciones con nuevos saberes circulantes en el campo educativo.

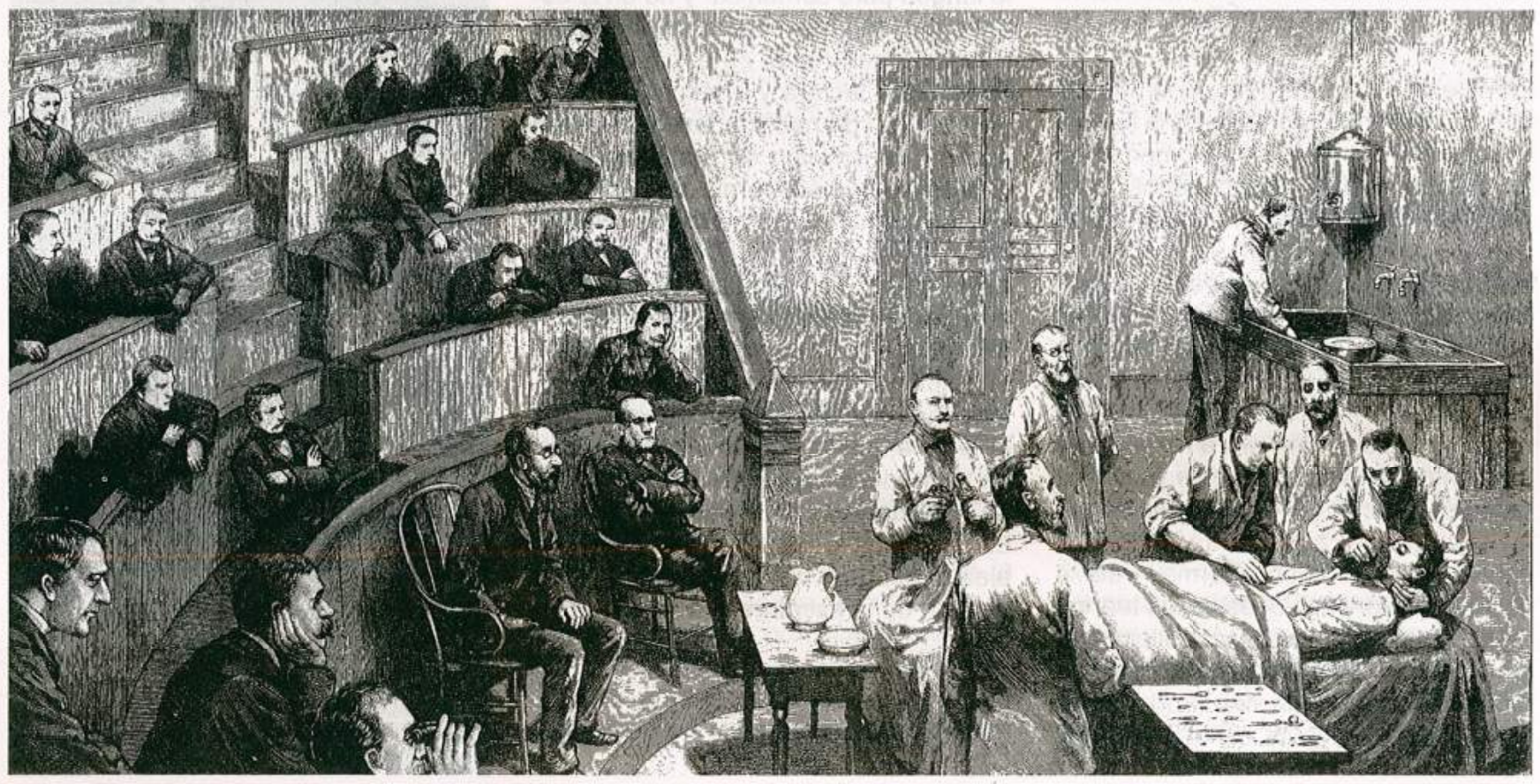




\section{REFERENCIAS}

Austin, J. L. (1982). Cómo hacer cosas con palabras, Barcelona: Paidós.

BaEnA, Luis Ángel. (1987). Lenguaje: comunicación y significación. Lenguaje. 16.

BARTHES, Roland. (1974). El grado cero de la escritura - Nuevos ensayos críticos-, México: Siglo XXI Editores.

Berger, P. y Luckman, N. (1968). La Construcción Social de la Realidad. Buenos Aires: Amorrortu.

Bobes, María Teresa. (1985). El diálogo, Madrid: Gredos.

BOYD, R. y RIGHERSON, P. (1985). Cultura y el Proceso de Evolución. Chicago: $U$. de Chicago.

BRUNER, Jerome. (1989). Realidad mental y mundos posibles. Barcelona: Gedisa.

BURón, J. (1993). Enseñar a aprender: introducción a la metacognición. Bilbao: Ediciones Mensajeros.

Cairney, T. H. (1992). Enseñanza de la comprensión lectora. Madrid: Ediciones.

CArdenas Paez, Alfonso. (1997). Hacia una pedagogía integral del lenguaje. Folios, 7 , pp. 33-42.

CArdenas Paez, Alfonso. (2001). Lenguaje, literatura y pedagogía de la lectura: Hacia una pedagogía del sentido. En Memorias, Segundo Congreso Colombiano de lectoescritura en lengua materna y lengua extranjera. Santa Fe de Bogotá: Universidad Distrital, pp 73-90.

Clavijo, Amparo. (2000). Formación de maestros: historia y vida. Bogotá: Plaza \& Janes.
Denzin, N. (1978). Introducción teórica al método sociológico. New Cork: McGrawHill.

Dubols, M. E. (1991). El proceso de la lectura: de la teoría a la práctica. Buenos Aires: Aique.

McEWAN, H. y KieRAn, E. (1998). La narrativa en la enseñanza, el aprendizaje y la investigación, Buenos Aires: Amorrurtu Editores.

FERREIRO, Emilia y TEBERosky, Ana. (1998). Los sistemas de escritura en el desarrollo en el niño. México: Siglo XXI Editores.

Giordan, André. (1987). Origenes del saber. Sevilla: Diada.

Goetz, J. y LECOMTE, M. (1998). Etnografia y diseño cualitativo en investigación educativa, Madrid: Morata.

Goodman, Kenneth. (1982). Lenguaje integral. Buenos Aires: Aique.

Habermas, Jurgen. (1989). Teoría de la acción comunicativa, Madrid: Cátedra

Larrosa, J., Arnaus, R., Ferrer, V. Pérez, N., Connelly, M., Clandinin, J. y Greene, M. (1995). Déjame que te cuente, Barcelona: Laertes.

Lineamientos curriculares Lengua casteIlana, Santa Fe de Bogotá: Ministerio de Educación Nacional.

LOPEZ, Gladys; CANON, Lilia y ARCINIEGAS, Esperanza (1997). Desarrollo de estrategias metacognitivas en la comprensión de texto académicos escritos a nivel universitario. Universidad del Valle.
LópEz Romo, H. (1998). La metodología de encuesta. En Técnicas de investigación: en sociedad, cultura y comunicación (Jesús Galindo Cáceres, comp.). México: Addison Wesley Longman.

Martínez, María. (1997). Discurso, proceso y significación. Cali: Univalle.

Moliner, Maria. (1991). Diccionario del uso del español. Madrid: Gredos.

Nıño, Hugo. (1978). Literatura de Colombia aborigen. En pos de la palabra, Bogotá: Instituto Colombiano de Cultura, p. 20.

ONG, Walter. (1982). Oralidad y escritura. Tecnología de la palabra. México: Fondo de Cultura Económica.

OrRantia, J., Sanchez, E. M. y Rosales, J (1990). Hacia una medición de las estrategias implicadas en el proceso de comprensión lectora. Lectura y vida, XI, 4.

RosenBLATT, L. M. (1978). Literatura como exploración. Buenos Aires: Aique.

SEARLE, J. (1969). Speechacts; an ensay in the philosophy of languaje. London Cambridge University Press.

TAYLOR, S. J. y BOGDAN, R. (2002). Introducción a los métodos cualitativos de investigación, Barcelona: Paidós.

SolE, I. (1994). Estrategias de Lectura Barcelona: Grao.

Van DiJk, Teun A. (1983). La ciencia del texto: un enfoque interdisciplinario. Barcelona: Paidós.

Zarzosa Escobedo, L. G. (1992). Repertorios básicos de comprensión de lectura. Lectura y vida, XIII, 1. 